OPEN ACCESS

Edited by:

Brian D. Lanoil,

University of Alberta, Canada

Reviewed by:

Ronald Oremland,

United States Geological Survey,

United States

Frank T. Robb,

University of Maryland, Baltimore,

United States

*Correspondence:

Gary M. King

gkingme@gmail.com

Specialty section: This article was submitted to Extreme Microbiology, a section of the journal

Frontiers in Microbiology

Received: 09 October 2017 Accepted: 11 December 2017 Published: 22 December 2017

Citation:

Myers MR and King GM (2017)

Perchlorate-Coupled Carbon Monoxide (CO) Oxidation: Evidence for a Plausible Microbe-Mediated

Reaction in Martian Brines.

Front. Microbiol. 8:2571.

doi: 10.3389/fmicb.2017.02571

\section{Perchlorate-Coupled Carbon Monoxide (CO) Oxidation: Evidence for a Plausible Microbe-Mediated Reaction in Martian Brines}

\author{
Marisa R. Myers and Gary M. King* \\ Department of Biological Sciences, Louisiana State University, Baton Rouge, LA, United States
}

The presence of hydrated salts on Mars indicates that some regions of its surface might be habitable if suitable metabolizable substrates are available. However, several lines of evidence have shown that Mars' regolith contains only trace levels of the organic matter needed to support heterotrophic microbes. Due to the scarcity of organic carbon, carbon monoxide (CO) at a concentration of about 700 parts per million (about $0.4 \mathrm{~Pa}$ ) might be the single most abundant readily available substrate that could support nearsurface bacterial activity. Although a variety of electron acceptors can be coupled to CO oxidation, perchlorate is likely the most abundant potential oxidant in Mars' brines. Whether perchlorate, a potent chaotrope, can support microbial CO oxidation has not been previously documented. We report here the first evidence for perchlorate-coupled CO oxidation based on assays with two distinct euryarchaeal extreme halophiles. CO oxidation occurred readily in $3.8 \mathrm{M} \mathrm{NaCl}$ brines with perchlorate concentrations from 0.01 to $1 \mathrm{M}$. Both isolates were able to couple $\mathrm{CO}$ with perchlorate or chlorate under anaerobic conditions with or without nitrate as an inducer for nitrate reductase, which serves as a perchlorate reductase in extreme halophiles. In the presence of perchlorate, $\mathrm{CO}$ concentrations were reduced to levels well below those found in Mars' atmosphere. This indicates that $\mathrm{CO}$ could contribute to the survival of microbial populations in hydrated salt formations or brines if water activities are suitably permissive.

\section{Keywords: carbon monoxide, extreme halophile, perchlorate reduction, Mars, chlorate reduction}

\section{INTRODUCTION}

The prospects for extraterrestrial life depend on two major factors: liquid water availability and the availability of reduced inorganic and organic substrates that can be used to sustain biochemical reactions (Brack et al., 2010). While discoveries of liquid water on Europa and Enceladus have addressed the former (Kargel et al., 2000; Zolotov et al., 2004; Zolotov, 2007; Waite et al., 2009; Ojha et al., 2014, 2015), much speculation remains about the latter. For Europa and Enceladus, simple organic and inorganic compounds have been identified as substrates for putative anaerobic processes (Chyba, 2000; Chyba and Phillips, 2002; McKay et al., 2008; Waite et al., 2017). Some of these substrates, especially hydrogen and formate, might be produced by serpentinization or other reactions beneath or associated with ice-capped oceans (Chyba, 2000). Evidence for liquid water has also been reported on Mars (Ojha et al., 2014, 2015), though Dundas et al. (2017) have 
indicated that volumes are likely low. Regardless, suites of organics at substrate level concentrations have not yet been identified in the regolith (Benner et al., 2000). Although hydrothermal activity and serpentinization might occur in the deep sub-surface of Mars, these processes are unlikely to contribute reactants to surface brines that might host microbes.

However, in contrast to Europa and Enceladus, Mars' atmosphere serves as a reservoir for carbon monoxide (CO), a relatively abundant [about 700 parts per million (ppm), $0.4 \mathrm{~Pa}$ ] photochemically produced reductant (Sindoni et al., 2010; Mahaffy et al., 2013) that can serve as a microbial metabolite under extreme conditions (King, 2015). Indeed, based on the total mass of Mars' atmosphere, its average molar mass (43.34 $\mathrm{g} \mathrm{mol}^{-1}$ ) and a concentration of $700 \mathrm{ppm}$, CO carbon occurs at $2.8 \mathrm{~mol} \mathrm{~m}^{-2}$ assuming a uniform distribution across the regolith surface. This is equivalent to the amount of carbon in $1 \mathrm{~m}^{3}$ of regolith with a density of $2 \mathrm{~g} \mathrm{~cm}^{-3}$ and a carbon concentration of $17 \mathrm{ppm}$. Since total organic carbon ranges from about 10 to $500 \mathrm{ppm}$ for meteorites derived from Mars (Steele et al., 2012) and Mars mudstones (Ming et al., 2014; Freissinet et al., 2015), CO might be the single most abundant readily available and renewable resource to drive metabolic activity.

Although diverse lineages of terrestrial bacteria can couple $\mathrm{CO}$ to multiple electron acceptors [e.g., molecular oxygen, nitrate, and sulfate (Ragsdale, 2004; King, 2006; King and Weber, 2007)], hydrated salts and brines on Mars are likely dominated by perchlorate and chlorate (Hecht et al., 2009; Kounaves et al., 2014; Clark and Kounaves, 2016), which have not been shown to support CO oxidation. However, both oxyanions support metabolism of various organic substrates by bacteria and archaea (Coates and Achenbach, 2004; Liebensteiner et al., 2013; Oren et al., 2014; Martinez-Espinosa et al., 2015; MehtaKolte et al., 2017) that reduce perchlorate via a dissimilatory perchlorate reductase (only bacteria to date) or a dissimilatory nitrate reductase (bacteria and archaea). To date, no COoxidizing dissimilatory perchlorate-reducing bacteria have been isolated, but numerous denitrifying and nitrate-respiring $\mathrm{CO}$ oxidizers have been reported (King, 2006, 2015; Weber and King, 2017). Results presented here provide the first evidence that some denitrifying and nitrate-respiring euryarchaeal extreme halophiles can couple $\mathrm{CO}$ oxidation in brines to perchlorate at concentrations up to $1 \mathrm{M}$.

\section{MATERIALS AND METHODS}

\section{Isolates}

Briefly, several denitrifying or nitrate-respiring CO-oxidizing extremely halophilic euryarchaeotes were obtained from salt crusts or soils in or near the Bonneville Salt Flats (BSF; Utah, United States). One of the denitrifiers, Haloarcula sp. PCN7, was obtained from enrichments initiated with BSF salt crusts $\left(40^{\circ} 45^{\prime} 26.4^{\prime \prime},-113^{\circ} 53^{\prime} 11.1^{\prime \prime}\right)$ while the nitrate-respiring Halobaculum sp. WSA2 was obtained from enrichments of a saline soil collected south of the BSF $\left(40^{\circ} 25^{\prime} 43.0^{\prime \prime},-114^{\circ}\right.$ $\left.00^{\prime} 55.4^{\prime \prime}\right)$. Enrichments were conducted in $160-\mathrm{ml}$ serum bottles under anoxic (nitrogen headspace with nitrate as an electron acceptor for Haloarcula sp. PCN7) or oxic conditions (for Halobaculum sp. WSA2) in medium CM1 (McDuff et al., 2016) with $25 \mathrm{mM}$ pyruvate. After addition of approximately 100 ppm CO (final concentration), serum bottles were incubated at $40^{\circ} \mathrm{C}$ with shaking. Headspace $\mathrm{CO}$ was monitored by gas chromatographic analysis (McDuff et al., 2016). Enrichments positive for $\mathrm{CO}$ oxidation were used to inoculate a series of bottles with fresh CM1 media, which were ultimately used to prepare dilutions that were spread onto CM1-pyruvate agar plates. Distinct colonies were selected and purified through repeated sub-culturing as necessary. Isolates were identified to genus by PCR amplification and sequencing of 16S rRNA genes obtained from genomic extracts using a MoBio Microbial DNA Extraction Kit (Folsom, CA, United States). PCR used an archaeal forward primer, Arch21F [5'-TTCCGGTTGATCCYGCCGGA$3^{\prime}$ (Delong, 1992)], and the universal reverse primer, 1492R [5'-CGGTTACCTTGTTACGACTT-3' (Lane, 1991)]. Purified amplicons (MoBio Ultraclean PCR Clean-Up Kit) were sequenced with an ABI 3130XL Genetic Analyzer at Louisiana State University. Sequences have been deposited in Genbank as accessions MF767880 and MF767881 for Haloarcula sp. PCN7 and Halobaculum sp. WSA2, respectively.

Genomic DNA extracts were also used to amplify the alpha sub-unit (narG) of nitrate reductase (Martinez-Espinosa et al., 2007, 2015) and the large sub-unit (coxL) of the form I CO dehydrogenase (King, 2003). Primers for narG were designed using nitrate reductase sequences found in the genomes of Haloarcula marismortui $43049^{\mathrm{T}}$, Haloferax mediterranei $33500^{\mathrm{T}}$, and other haloarchaea represented in the Integrated Microbial Genomes resource ${ }^{1}$. Primers pNar1F (5'-ACGAYTGGTAYCACAACGAC-3') and pNar1R $\left(5^{\prime}\right.$-AGTTCSAGRWACCAGTCGTG-3') yield products approximately $990 \mathrm{bp}$ in size. Details of coxL PCR have been published previously (King, 2003); PCR primers used were archcoxF (5'-GGYGGSTTYGGSAASAAGGT-3') and PSr (5'-YTCGAYGATCATCGGRTTGA-3'). Amplicons obtained using these primers were sequenced bi-directionally as above. Phylogenetic analyses of inferred amino acid sequences for coxL and narG were conducted using MEGA7 (Kumar et al., 2015) using a neighbor-joining algorithm and a Poisson correction; all gapped positions were deleted. CoxL sequences have been deposited in Genbank as accessions MF773971 and MF773972 for Haloarcula sp. PCN7 and Halobaculum sp. WSA2, respectively, and as MF773973 and MF773974 for the respective narG genes.

Assays for nitrate respiration and denitrification were conducted using API 20NE test strips (bioMérieux S.A., Marcy l'Etoile, France) according to the manufacturer's instructions. Confirmation of the results for Haloarcula sp. PCN7 was obtained by incubating the isolate in sealed $10-\mathrm{cm}^{3}$ syringes with CM1 medium containing pyruvate and nitrate. Under these conditions, copious production of gas bubbles presumed to be dinitrogen was interpreted as evidence for denitrification.

Although both Haloarcula sp. PCN7 and Halobaculum sp. WSA2 can utilize $\mathrm{CO}$ at concentrations in excess of $100 \mathrm{ppm}$,

\footnotetext{
${ }^{1}$ https://img.jgi.doe.gov
} 
growth was not observed for either isolate. This is consistent with results obtained for other CO-oxidizing extreme halophiles (McDuff et al., 2016).

\section{Perchlorate-Coupled CO Oxidation}

Haloarcula sp. PCN7 and Halobaculum sp. WSA2 were assessed for their ability to couple CO oxidation to perchlorate reduction using stationary phase cells first grown aerobically in $3.8 \mathrm{M}$ CM1-pyruvate medium to provide a suitable level of cell biomass. Since CO uptake is typically induced by substrate limitation during stationary phase, it was not necessary to preincubate cells with CO. To initiate assays, cells were harvested by centrifugation, washed in $3.8 \mathrm{M} \mathrm{CM1}$ without pyruvate, and resuspended into $3.8 \mathrm{M} \mathrm{CM1}$ with or without $2.5 \mathrm{mM}$ pyruvate to support basal metabolism; treatments included the following: aerobic incubation with no perchlorate; anaerobic incubation (nitrogen headspace) with $0.01 \mathrm{M}$ perchlorate with or without $0.25 \mathrm{mM}$ nitrate; anaerobic incubation with no electron acceptor and anaerobic incubation of autoclaved cells with $0.01 \mathrm{M}$ perchlorate. Resuspended cells $(10 \mathrm{ml}$, $A_{600} \sim 0.4$ ) were incubated in triplicate $160-\mathrm{ml}$ serum bottles sealed with neoprene rubber stoppers. $\mathrm{CO}$ was added to a final headspace $(150 \mathrm{ml})$ concentration of $\sim 10 \mathrm{ppm}$ (about $1 \mathrm{~Pa})$. Headspace CO concentrations were assayed periodically by removing sub-samples for analysis by gas chromatography. Anoxic treatments contained resazurin to monitor oxygen contamination.

To assess tolerance of elevated perchlorate concentrations cells were grown to stationary phase, centrifuged, and washed as described above. Resuspended cells were aliquoted into $160-\mathrm{ml}$ serum bottles to which either $0.01,0.1$, or $1 \mathrm{M}$ perchlorate was added; each perchlorate treatment was incubated with aerobic (21\% oxygen) or anoxic (flushed with nitrogen) headspaces. Experimental treatments without perchlorate included oxic, no electron acceptor, and an autoclaved kill control. All treatments were performed in triplicate.

Perchlorate concentrations were determined periodically during selected treatments using sub-samples obtained by needle and syringe. Perchlorate concentrations were measured with an ion-selective electrode (Thomas Scientific, Swedesboro, NJ, United States), standardized with perchlorate dissolved in growth medium. Total assay volume was $5 \mathrm{ml}$ including $500 \mu 1$ of culture and $100 \mu \mathrm{l}$ of $1 \mathrm{M}$ sodium acetate as an ionic strength adjustment buffer. A 10-fold sample dilution was required due to the high $\mathrm{NaCl}$ concentrations in the media.

Chlorate concentrations were monitored via a colorimetric O-tolidine assay (Couture, 1998). O-Tolidine assays contained in a 1-ml final reaction mixture: $4 \mu \mathrm{l}$ sample, $396 \mu \mathrm{l}$ deionized water, $100 \mu \mathrm{l} \mathrm{O}$-tolidine, and $500 \mu \mathrm{l}$ concentrated $(12 \mathrm{M}) \mathrm{HCl}$. After $10 \mathrm{~min}$ of incubation, absorbance was read at $448 \mathrm{~nm}$.

Water potentials of growth media were assessed using a WP4-T water potential meter [Decagon Devices, Pullman, WA, United States (Weber and King, 2009)]. Water potential (with units of pressure, e.g., MPa) is a measure of the chemical potential of water molecules in solutions, and varies with temperature and solute concentration and composition (King, 2017). Pure water has a potential of 0 while solutions have potentials $<0$ with decreasing values representing increasing physiological water stress. For reference, seawater containing 3.5\% $\mathrm{NaCl}$ has a water potential of about $-2.8 \mathrm{MPa}$ and $\mathrm{NaCl}$ saturated brines have potentials about $-40 \mathrm{MPa}$ (King, 2015). The water potentials of CM1 media with varied perchlorate concentration were measured to account for variations in water stresses.

\section{RESULTS AND DISCUSSION}

\section{Perchlorate Linked CO Oxidation by a Denitrifying Extreme Halophile}

To assess the feasibility of CO-coupled perchlorate reduction in brines, denitrifying and nitrate-respiring extremely halophilic enrichments and isolates were obtained from the BSF and nearby saline soils (UT, United States). Previous reports have shown that a dissimilatory periplasmic nitrate reductase catalyzes perchlorate, chlorate, and nitrate reduction in the denitrifying extreme halophile, Hfx. mediterranei $33500^{\mathrm{T}}$, with chlorate reduction rates exceeding those for nitrate (Martinez-Espinosa et al., 2015). However, there has been no evidence to date for dissimilatory perchlorate-reducing extreme halophiles.

Haloarcula sp. PCN7 was selected as a model denitrifier for assays with perchlorate. It oxidizes $\mathrm{CO}$ in media with up to $5.2 \mathrm{M}$ $\mathrm{NaCl}$ (halite saturation) using molecular oxygen as an electron acceptor; it also oxidizes $\mathrm{CO}$ using nitrate during denitrification. It possesses a canonical molybdenum-dependent form I CO dehydrogenase (Supplementary Figure 1), and it contains a nitrate reductase gene as established by PCR amplification of the narGH structural gene and genomic analysis. Sequence and phylogenetic analyses have revealed characteristic motifs for dissimilatory nitrate reductases (Martinez-Espinosa et al., 2007) and a nucleotide identity of $80.3 \%$ with the Hfx. mediterranei $33500^{\mathrm{T}}$ narGH gene (Supplementary Figure 2).

Since CO uptake was typically assayed with initial headspace concentrations of about $10 \mathrm{ppm}$ with $10 \mathrm{ml}$ of medium and a headspace of $150 \mathrm{~cm}^{3}$, equivalent to only about $60 \mathrm{nmol}$ total, CO-coupled perchlorate reduction could not be observed directly by analyses of changes in perchlorate concentrations in hypersaline media. Under the assay conditions, complete CO oxidation would have resulted in a maximum perchlorate decrease of only $1.5 \mu \mathrm{M}$ out of $0.01 \mathrm{M}$ assuming the following stoichiometries with chlorite as the end-product:

$$
\begin{aligned}
& \mathrm{CO}+\mathrm{ClO}_{4}^{-} \rightarrow \mathrm{CO}_{2}+\mathrm{ClO}_{3}^{-} \\
& \mathrm{CO}+\mathrm{ClO}_{3}^{-} \rightarrow \mathrm{CO}_{2}+\mathrm{ClO}_{2}^{-}
\end{aligned}
$$

Therefore, coupling of $\mathrm{CO}$ oxidation to perchlorate was established by comparing $\mathrm{CO}$ uptake in anaerobic assays with no electron acceptors to uptake in aerobic assays (air headspaces), and anaerobic assays with perchlorate only $(0.01 \mathrm{M})$, nitrate only $(0.25$ or $25 \mathrm{mM})$, and perchlorate $(0.01 \mathrm{M})$ with a low concentration of nitrate $(0.25 \mathrm{mM})$ as a nar gene inducer.

Under these conditions, $\mathrm{CO}$ uptake occurred at rates of 3.0-6.5 nmol [mg protein $]^{-1} \mathrm{~d}^{-1}$, but only in the presence of 

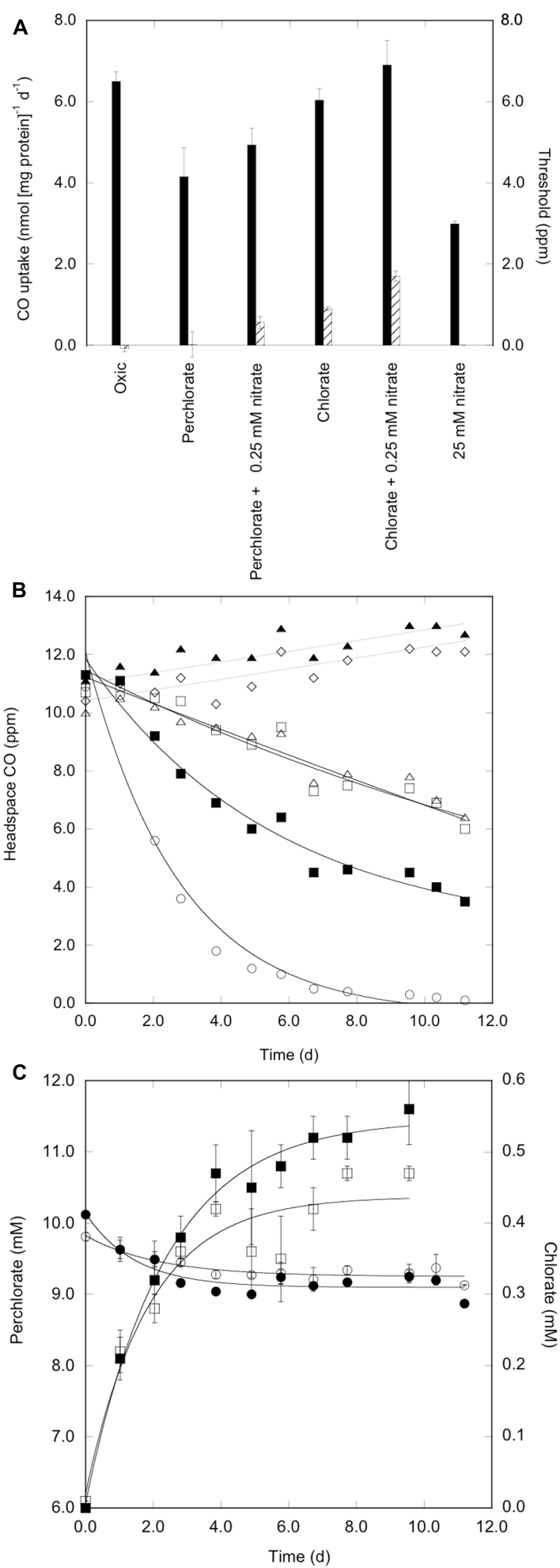

FIGURE 1 | Perchlorate-coupled CO uptake by Haloarcula sp. PCN7. (A) CO uptake rates (nmol CO [mg protein] $]^{-1} \mathrm{~d}^{-1}$; solid bars) and uptake thresholds (ppm; cross-hatched bars) for isolate Haloarcula sp. PCN7 during varied oxic and anoxic incubations in $3.8 \mathrm{M} \mathrm{NaCl}$. (B) Headspace CO versus time for isolate Haloarcula sp. PCN7 during varied oxic and anoxic incubations in

(Continued)
FIGURE 1 | Continued

3.8 M NaCl; oxic, $\bigcirc$; perchlorate plus nitrate, $\mathbf{\square}$; perchlorate, $\square$; $25 \mathrm{mM}$ nitrate, $\Delta ; 0.25 \mathrm{mM}$ nitrate, $\mathbf{\Delta}$; no electron acceptor, $\diamond$. (C) Perchlorate uptake (circles) and chlorate production (squares) for perchlorate only (open symbols) and perchlorate plus nitrate (closed symbols) during assays with isolate Haloarcula sp. PCN7 in media with $2.5 \mathrm{mM}$ pyruvate. All values are means of triplicate cell suspensions \pm 1 standard error. Negative threshold values were not statistically different than zero.

oxygen, perchlorate with or without $0.25 \mathrm{mM}$ nitrate, or nitrate at $25 \mathrm{mM}$; no uptake was observed with $0.25 \mathrm{mM}$ nitrate alone, without electron acceptors or in killed controls (Figures 1A,B). In the low nitrate treatment, nitrate was likely unavailable for $\mathrm{CO}$ oxidation due to rapid consumption during basal metabolism. Initial $\mathrm{CO}$ uptake rates in perchlorate and nitrate treatments were similar, and significantly lower than rates in aerobic assays (Figures 1A,B). Although treatments with $0.01 \mathrm{M}$ perchlorate plus $0.25 \mathrm{mM}$ nitrate yielded a small increase in uptake relative to perchlorate only (Figure 1A), the effect was not significant and varied among trials. These observations differ from those for $H f x$. mediterranei $33500^{\mathrm{T}}$, which reduced perchlorate and chlorate only after growth in anaerobic media with nitrate (Martinez-Espinosa et al., 2015). This indicates that synthesis of dissimilatory nitrate reductase might be induced by anoxia alone in some denitrifying extreme halophiles as has been observed for some bacteria [e.g., Achromobacter cycloclastes (Coyne and Tiedje, 1990)]. Although nitrate is likely present in Mars' regolith (Kounaves et al., 2014; Stern et al., 2017), results from Haloarcula sp. PCN7 suggest that it might not be required to initiate perchlorate reduction in brines.

Threshold levels for CO uptake did not vary consistently among aerobic, perchlorate, and nitrate treatments (Figure 1A). Nonetheless, CO uptake with each of the electron acceptors continued at levels comparable to or less than ambient terrestrial atmospheric values $(0.2-0.4 \mathrm{ppm}$, about $0.02-0.04 \mathrm{~Pa})$, which are far below those in Mars' atmosphere. This suggests that $\mathrm{CO}$ uptake need not be confined to Mars' surface regolith, but that it could occur in sub-surface horizons that might provide more favorable physical conditions (e.g., lower UV/ionizing radiation exposure).

Results from a separate assay were similar, but by including $2.5 \mathrm{mM}$ pyruvate in the incubation medium as catabolic substrate, it was possible to observe perchlorate reduction

TABLE 1 | CO uptake rate constants $\left(\mathrm{d}^{-1}\right)$ and uptake thresholds (ppm) for Haloarcula sp.

\begin{tabular}{lcc}
\hline Treatment & Rate constant & Threshold \\
\hline Aerobic & $0.506(0.034)^{\mathrm{a}}$ & $0.08(0.05)^{\mathrm{a}}$ \\
9:1 perchlorate:chlorate & $0.389(0.009)^{\mathrm{b}}$ & $1.67(0.11)^{\mathrm{b}}$ \\
1:9 perchlorate:chlorate & $0.568(0.023)^{\mathrm{a}}$ & $3.75(0.25)^{\mathrm{c}}$
\end{tabular}

PCN7 incubated under aerobic conditions or with anoxic conditions and varied ratios of perchlorate and chlorate concentrations, concentrations in millimolar. All values are means ( \pm 1 standard error) of triplicate determinations. Statistically significant differences based on analysis of variance with a Bonferroni post hoc test are indicated by superscripts. 


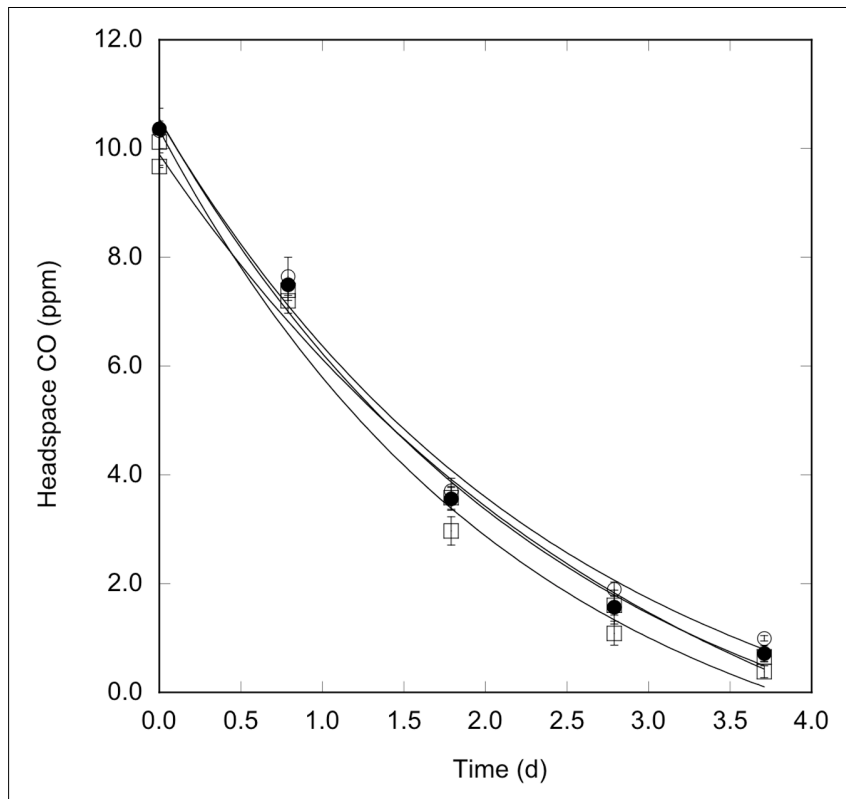

FIGURE 2 | Perchlorate tolerance by Haloarcula sp. PCN7 under oxic conditions. CO uptake by Haloarcula sp. PCN7 during aerobic incubations with no perchlorate $(\bigcirc)$ or $0.01(\bullet), 0.1(\square)$, or $1 \mathrm{M}(\mathbf{\square})$ perchlorate. All values are means of triplicate cell suspensions for \pm 1 standard error.

and chlorate formation (Figure 1C). During the period of maximum $\mathrm{CO}$ oxidation, perchlorate decreased by approximately $1 \mathrm{mM}$, while chlorate increased by about $0.5 \mathrm{mM}$ (Figure 1C); chlorite formation from chlorate might have accounted for part or all of the difference. The decrease in perchlorate and increase in chlorate concentrations were greatest in the presence of $0.25 \mathrm{mM}$ nitrate (Figure 1C), but perchlorate reduction did not require nitrate, which was consistent with results from $\mathrm{CO}$ oxidation. The fact that $\mathrm{CO}$ uptake did not occur in the absence of an electron acceptor (e.g., perchlorate or $25 \mathrm{mM}$ nitrate), but occurred while perchlorate was reduced and chlorate was formed confirmed the potential for CO-coupled perchlorate reduction by extreme halophiles.

\section{Chlorate-Coupled CO Oxidation}

Carbon monoxide uptake was also observed with chlorate, an intermediate in the perchlorate reduction pathway (Coates and Achenbach, 2004). CO uptake rates with chlorate were comparable to uptake rates with oxygen and exceeded uptake rates with perchlorate (Figure 1A), a pattern consistent with observations of chlorate and perchlorate reduction by Hfx. mediterranei $35000^{\mathrm{T}}$ (Martinez-Espinosa et al., 2015). However, CO uptake threshold values with chlorate were significantly higher than with other oxidants (Figure 1A), which indicated that the relative abundance of perchlorate and chlorate could affect $\mathrm{CO}$ uptake rates when both co-occur. This possibility was confirmed by comparing results from assays with 9:1 and 1:9 $\mathrm{mM}$ perchlorate:chlorate ratios, respectively (Table 1 and Supplementary Figure 3). In agreement

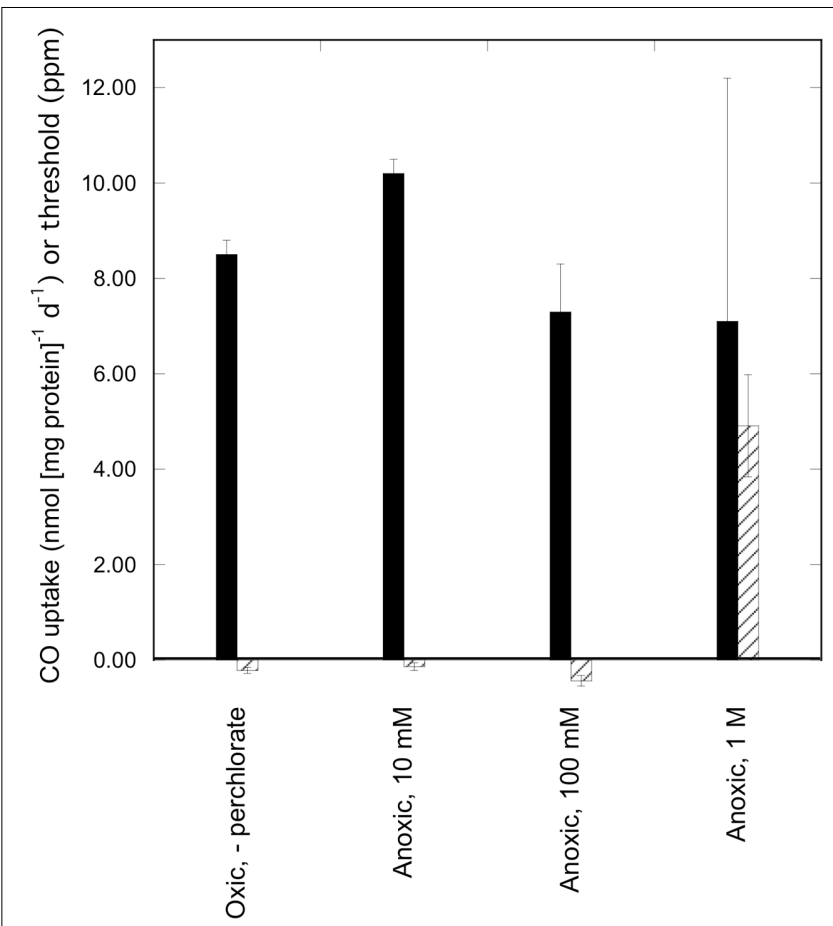

FIGURE 3 | Perchlorate tolerance by Haloarcula sp. PCN7 under anoxic conditions. CO uptake rates (nmol CO [mg protein $]^{-1} \mathrm{~d}^{-1}$; solid bars) and uptake thresholds (ppm; cross-hatched bars) for isolate Haloarcula sp. PCN7 during aerobic and anaerobic incubations in $3.8 \mathrm{M} \mathrm{NaCl}$ with varied concentrations of perchlorate; values are means of triplicate cell suspensions \pm 1 standard error; negative threshold values were not significantly different than zero.

with prior assays, $\mathrm{CO}$ uptake rate constants with 9:1 $\mathrm{mM}$ chlorate:perchlorate were greater than uptake rate constants with 1:9 $\mathrm{mM}$ perchlorate:chlorate. On the other hand, the addition of $1 \mathrm{mM}$ chlorate to $9 \mathrm{mM}$ perchlorate yielded CO uptake thresholds $(1.67 \mathrm{ppm})$ substantially greater than values observed for perchlorate alone or perchlorate plus $0.25 \mathrm{mM}$ nitrate; thresholds were even higher with $9 \mathrm{mM}$ chlorate plus $1 \mathrm{mM}$ perchlorate (Table 1). Nonetheless, all uptake threshold values were lower than ambient atmospheric levels on Mars, and thus would permit surface and sub-surface CO uptake.

\section{Response to Elevated Perchlorate Concentrations}

Although CO uptake assays were typically conducted with $0.01 \mathrm{M}$ perchlorate, additional assays explored activity at concentrations up to $1 \mathrm{M}$. No differences in $\mathrm{CO}$ uptake rate constants or thresholds were observed when Haloarcula sp. PCN7 was incubated with ambient air and $0-1 \mathrm{M}$ perchlorate in a brine medium with a water potential of $-19 \mathrm{MPa}$ (Figure 2). Oxic incubations facilitated comparisons with some prior studies. For example, Oren et al. (2014) found no effect of $0.2 \mathrm{M}$ perchlorate on aerobic growth by several archaeal extreme halophiles, but noted partial to substantial inhibition at higher concentrations 


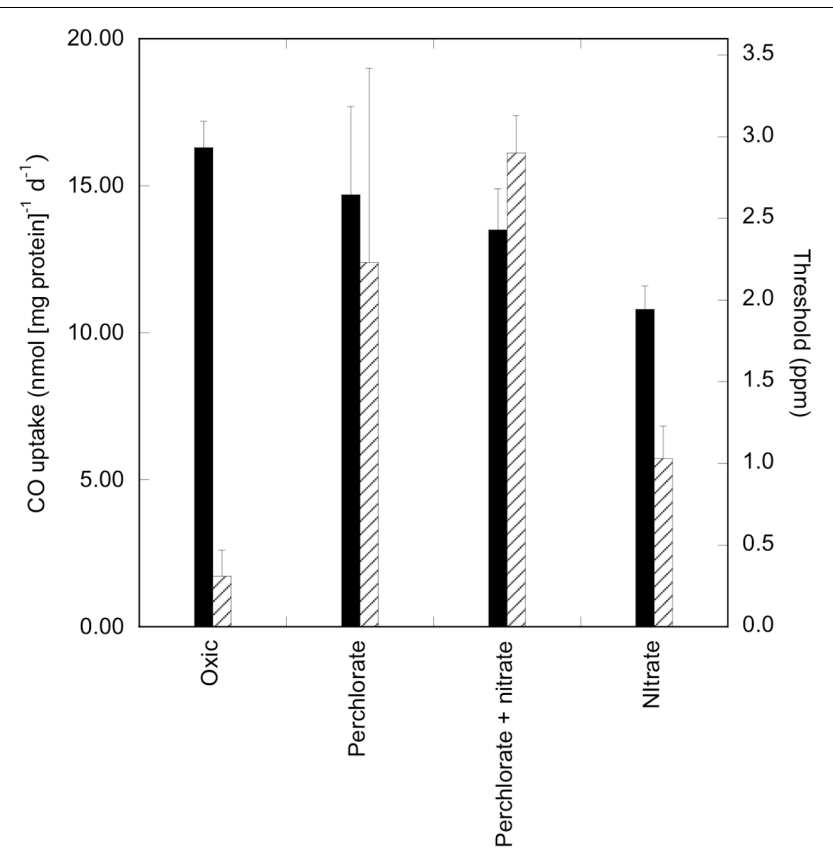

FIGURE 4 | Perchlorate-coupled CO uptake by Halobaculum sp. WSA2. CO uptake rates (nmol CO [mg protein] $]^{-1} \mathrm{~d}^{-1}$ ) and uptake thresholds (ppm) for isolate Halobaculum sp. WSA2 during aerobic and anaerobic incubations in $3.8 \mathrm{M} \mathrm{NaCl}$ with perchlorate, perchlorate $+0.25 \mathrm{mM}$ nitrate, or with $25 \mathrm{mM}$ nitrate; values are means of triplicate cell suspensions \pm 1 standard error

(0.4-0.6 M). Limited tolerance of $1 \mathrm{M}$ perchlorate has been also reported for several bacterial isolates during aerobic growth assays (Al Soudi et al., 2017), but those assays were conducted at much higher water potentials $(-1.4$ to $-5.6 \mathrm{MPa}$, e.g., moderate salt concentrations and less physiological water stress) than used in this study $(-19 \mathrm{MPa})$. Thus, the ability to oxidize $\mathrm{CO}$ during substantial water stress (i.e., lower water potentials) and simultaneously high concentrations of a potent chaotrope [perchlorate (Cray et al., 2013)] is unprecedented.

Haloarcula sp. PCN7 was also able to couple CO uptake with elevated perchlorate concentrations (up to $1 \mathrm{M}$ ) under anaerobic conditions. Anaerobic perchlorate tolerance at such high levels has not been previously documented, although $0.05 \mathrm{M}$ perchlorate did not affect $\mathrm{CO}$ oxidation by Alkalilimnicola ehrlichii MLHE-1 (King, 2015), which was unable to use it as an electron acceptor. For Haloarcula sp. PCN7 CO uptake rates for $0.01,0.1$, and $1 \mathrm{M}$ treatments did not differ statistically, but uptake rates with $1 \mathrm{M}$ perchlorate were more variable (Figure 3) and threshold concentrations were significantly higher $(4.9 \pm 1.0 \mathrm{ppm}$ ) than for 0.01 and $0.1 \mathrm{M}$ perchlorate (not statistically different than zero). Differences in thresholds with $1 \mathrm{M}$ perchlorate could reflect lower water potentials in the assay media $(-24.5,-25.4$, and $-32.6 \mathrm{MPa}$ for $0.01,0.1$, and $1 \mathrm{M}$, respectively). Collectively, these results indicated that perchlorate was not only tolerated at high concentrations under anaerobic conditions, but that it was exploited as an oxidant. This in turn supports the possibility that high perchlorate concentrations in extraterrestrial brines could sustain microbial life, potentially including biotypes specifically adapted for perchlorate dissimilation.

\section{Perchlorate-CO Coupling by Nitrate-Respiring Extreme Halophiles}

Coupling of $\mathrm{CO}$ oxidation with perchlorate reduction was confirmed with a second isolate, Halobaculum sp. WSA2. Like Haloarcula sp. PCN7, Halobaculum sp. WSA2 possesses a form I molybdenum-dependent $\mathrm{CODH}$ and a dissimilatory nitrate reductase (Supplementary Figures 1, 2). However, unlike Haloarcula sp. PCN7, Halobaculum sp. WSA2 produces nitrite as a terminal product from nitrate reduction, and does not possess genes for nitrite or nitrous oxide reduction based on initial results of a genome analysis. It oxidized $\mathrm{CO}$ under aerobic conditions, or with nitrate, perchlorate, or perchlorate plus nitrate at rates similar to those for Haloarcula sp. PCN7 and with similar threshold values (Figure 4).

The ability of Halobaculum sp. WSA2 to reduce perchlorate is notable, because a nitrate-respiring gammaproteobacterium, $A$. ehrlichii MLHE-1, could not do so although it readily oxidized CO with nitrate (King, 2015). This might reflect a fundamental difference in the location of the nitrate reductase active site in the narGH enzyme system of haloarchaea versus that of some bacteria (Martinez-Espinosa et al., 2007). In particular, the haloarchaeal narG active site has been described as periplasmic (Martinez-Espinosa et al., 2007), which could result in greater accessibility to perchlorate than a cytoplasmic orientation in bacteria does. Thus, only a sub-set of CO-oxidizing nitrate respirers may reduce perchlorate, and a similar constraint might apply to denitrifiers.

In summary, this study demonstrates for the first time that perchlorate and chlorate can be coupled as electron acceptors to anaerobic $\mathrm{CO}$ oxidation in $\mathrm{NaCl}$ brines by diverse euryarchaeal extreme halophiles capable of denitrification or nitrate respiration. Under anoxic conditions, perchlorate concentrations from 0.01 to $1 \mathrm{M}$ in $3.8 \mathrm{M} \mathrm{NaCl}$ with or without nitrate supported $\mathrm{CO}$ oxidation. Oxidation occurred at $\mathrm{CO}$ concentrations comparable to those in Mars' atmosphere and at concentrations comparable to or lower than those in Earth's atmosphere.

The ubiquity of CO in the cosmos (Dickman, 1978), its presence at relatively high concentrations in the solar system (Elsila et al., 1997; Greaves et al., 2011; Materese et al., 2015), and the possibility of coupling $\mathrm{CO}$ oxidation to diverse electron acceptors (Ragsdale, 2004; King, 2006; King and Weber, 2007) suggest that CO-based metabolism could fuel microbial activity in some of the exoplanetary systems that have been discovered to date. Evidence for high perchlorate concentrations on Mars and results from this study also indicate that $\mathrm{CO}$ could fuel metabolism by either relict or introduced extreme halophiles in hydrated salts or brines (Ojha et al., 2015).

\section{AUTHOR CONTRIBUTIONS}

MM isolated and characterized strains, conducted growth, CO, and chemical assays, and contributed to 
manuscript development. GK conceived the study, conducted fieldwork and sample collections for isolate enrichments, contributed to experimental analyses, and wrote the manuscript.

\section{FUNDING}

This work was supported in part by the National Science Foundation EAR-1565499 and NASA awards NNX15AE82A and 15-EXO15_2-0147.

\section{REFERENCES}

Al Soudi, A. F., Farhat, O., Chen, F., Clark, B. C., and Schneegurt, M. A. (2017). Bacterial growth tolerance to concentrations of chlorate and perchlorate relevant to Mars. Int. J. Astrobiol. 16, 229-235. doi: 10.1017/ S1473550416000434

Benner, S. A., Devine, K. G., Matveeva, L. N., and Powell, D. H. (2000). The missing organic molecules on Mars. Proc. Natl. Acad. Sci. U.S.A. 97, 2425-2430. doi: 10.1073/pnas.040539497

Brack, A., Horneck, G., Cockell, C. S., Bérces, A., Belisheva, N. K., Eiroa, C., et al. (2010). Origin and evolution of life on terrestrial planets. Astrobiology 10, 69-76. doi: 10.1089/ast.2009.0374

Chyba, C. F. (2000). Energy for microbial life on Europa. Nature 403, 381-382. doi: $10.1038 / 35000281$

Chyba, C. F., and Phillips, C. B. (2002). Europa as an abode of life. Orig. Life Evol. Biosph. 32, 47-68. doi: 10.1023/A:1013958519734

Clark, B. C., and Kounaves, S. P. (2016). Evidence for the distribution of perchlorates on mars. Int. J. Astrobiol. 15, 311-318. doi: 10.1017/ S1473550415000385

Coates, J. D., and Achenbach, L. A. (2004). Microbial perchlorate reduction: rocketfuelled metabolism. Nature Rev. Microbiol. 2, 569-580. doi: 10.1038/nrmicro926

Couture, E. (1998). Chlorate, and Chlorite Analysis in Seawater, Chlorate Sinks. MS thesis, Dalhousie University, Halifax, NS.

Coyne, M. S., and Tiedje, J. M. (1990). Induction of denitrifying enzymes in oxygenlimited Achromobacter cycloclastes continuous culture. FEMS Microbiol. Ecol. 6, 263-270. doi: 10.1111/j.1574-6968.1990.tb03949.x

Cray, J. A., Russell, J. T., Timson, D. J., Singhal, R. S., and Hallsworth, J. E. (2013). A universal measure of chaotropicity and kosmotropicity. Environ. Microbiol. 15, 287-296. doi: 10.1111/1462-2920.12018

Delong, E. F. (1992). Archaea in coastal marine environments. Proc. Natl. Acad. Sci. U.S.A. 89, 5685-5689. doi: 10.1073/pnas.89.12.5685

Dickman, R. L. (1978). The ratio of carbon monoxide to molecular hydrogen in interstellar dark clouds. Astrophys. J. Suppl. Ser. 37, 407-427. doi: 10.1086/ 190535

Dundas, C. M., McEwen, A. S., Chojnacki, M., Milazzo, M. P., Byrne, S., McElwaine, J. N., et al. (2017). Granular flows at recurring slope lineae on Mars indicate a limited role for liquid water. Nature Geosci. 10, 903-907. doi: 10.1038/s41561-017-0012-5

Elsila, J., Allamandola, L. J., and Sandford, S. A. (1997). The $2140 \mathrm{~cm}^{-1}$ (4.673 microns) solid CO band: the case for interstellar O2 and N2 and the photochemistry of nonpolar interstellar ice analogs. Astrophys. J. 479, 818-838. doi: $10.1086 / 303906$

Freissinet, C., Glavin, D. P., Mahaffy, P. R., Miller, K. E., Eigenbrode, J. L., Summons, R. E., et al. (2015). Organic molecules in the sheepbed mudstone, gale crater, mars. J. Geophys. Res. Planets 120, 495-514. doi: 10.1002/ 2014JE004737

Greaves, J. S., Helling, Ch., and Friberg, P. (2011). Discovery of carbon monoxide in the upper atmosphere of pluto. Mon. Not. R. Astron. Soc. 414, L36-L40. doi: 10.1111/j.1745-3933.2011.01052.x

Hecht, M. H., Kounaves, S. P., Quinn, R. C., West, S. J., Young, S. M., Ming, D. W., et al. (2009). Detection of perchlorate and the soluble chemistry of martian soil at the phoenix lander site. Science 325, 64-67. doi: 10.1126/science. 1172466

\section{ACKNOWLEDGMENTS}

The authors thank Dr. B. Bebout for helpful discussions and collaboration, and Ms. C. Ruiz for technical support.

\section{SUPPLEMENTARY MATERIAL}

The Supplementary Material for this article can be found online at: https://www.frontiersin.org/articles/10.3389/fmicb. 2017.02571/full\#supplementary-material

Kargel, J. S., Kay, J. Z., Head, J. W., Marion, G. M., Sassen, R., Crowley, J. K., et al. (2000). Europa's crust and ocean: origin, composition and the prospects for life. Icarus 148, 226-265. doi: 10.1006/icar.2000.6471

King, G. M. (2003). Molecular and culture-based analyses of aerobic carbon monoxide oxidizer diversity. Appl. Environ. Microbiol. 69, 7257-7265. doi: 10.1128/AEM.69.12.7257-7265.2003

King, G. M. (2006). Nitrate-dependent anaerobic oxidation of carbon monoxide oxidation by aerobic CO-oxidizing bacteria. FEMS Microbiol. Ecol. 56, 1-7. doi: 10.1111/j.1574-6941.2006.00065.x

King, G. M. (2015). Carbon monoxide as a metabolic energy source for extremely halophilic microbes: implications for microbial activity in mars regolith. Proc. Natl. Acad. Sci. U.S.A. 112, 4465-4470. doi: 10.1073/pnas.1424989112

King, G. M. (2017). "Water potential as a master variable for atmosphere-soil trace gas exchange in arid and semi-arid ecosystems" in The Biology of Arid Soils, ed. S. Blaire (Berlin: Walter de Gruyter GmbH), 31-45.

King, G. M., and Weber, C. F. (2007). Distribution, diversity and ecology of aerobic CO-oxidizing bacteria. Nat. Rev. Microbiol. 5, 107-118. doi: 10.1038/ nrmicro1595

Kounaves, S. P., Carrier, B. L., O’Neil, G. D., Stroble, S. T., and Claire, M. W. (2014). Evidence of martian perchlorate, chlorate and nitrate in mars meteorite EETA79001: implications for oxidants and organics. Icarus 229, 1-22. doi: 10.1016/j.icarus.2013.11.012

Kumar, S., Stecher, G., and Tamura, K. (2015). MEGA7: molecular evolutionary genetics analysis version 7.0 for bigger datasets. Mol. Biol. Evol. 33, 1870-1874. doi: $10.1093 / \mathrm{molbev} / \mathrm{msw} 054$

Lane, D. J. (1991). "16S/23S rRNA sequencing," in Nucleic Acid Techniques in Bacterial Systematics, eds E. Stackebrandt, and M. Goodfellow (Hoboken, NJ: John Wiley \& Sons), 115-175.

Liebensteiner, M. G., Pinske, M. W. H., Schaap, P. J., Stams, A. J. M., and Lomans, B. P. (2013). Archaeal (per)chlorate reduction at high temperature: an interplay of biotic and abiotic reactions. Science 340, 85-87. doi: 10.1126/science.1233957

Mahaffy, P. R., Webster, C. R., Atreya, S. K., Franz, H., Wong, M., Conrad, P. G., et al. (2013). Abundance and isotopic composition of gases in the martian atmosphere from the curiosity rover. Science 341, 263-266. doi: $10.1126 /$ science. 1237966

Martinez-Espinosa, R. M., Dridge, E. J., Bonete, M. J., Butt, J. N., Butler, C. S., Sargent, F., et al. (2007). Look on the positive side! The orientation, identification and bioenergetics of 'Archaeal'membranebound nitrate reductases. FEMS Microbiol. Lett. 276, 129-139. doi: 10.1111/j.1574-6968.2007.00887.x

Martinez-Espinosa, R. M., Richardson, D. J., and Bonete, M. J. (2015). Characterization of chlorate reduction in the haloarchaeon Haloferax mediterranei. Biochem. Biophys. Acta 1850, 587-594. doi: 10.1016/j.bbagen. 2014.12.011

Materese, C. K., Cruikshank, D. P., Sandford, S. A., Imanaka, H., and Nuevo, M. (2015). Ice chemistry on outer solar system bodies: electron radiolysis of N2-, CH4-, and CO-containing ices. Astrophys. J. 812, 150-159. doi: 10.1088/0004$637 \mathrm{X} / 812 / 2 / 150$

McDuff, S., King, G. M., Neupane, S., and Myers, M. R. (2016). Isolation and characterization of extremely halophilic CO-oxidizing euryarchaeota from hypersaline cinder, sediments and soils and description of a novel CO oxidizer, Haloferax namakaokahaiae Mke2.3. FEMS Microbiol. Ecol. 92:fiw028. doi: 10.1093/femsec/fiw028 
McKay, C. P., Porco, C. C., Altheide, T., Davis, W. L., and Kral, T. A. (2008). The possible origin and persistence of life on Enceladus and detection of biomarkers in the plume. Astrobiology 8, 909-919. doi: 10.1089/ast.2008.0265

Mehta-Kolte, M. G., Loutey, D., Wang, O., Youngblut, M. D., Hubbard, C. G., Wetmore, K. M., et al. (2017). Mechanism of H2S oxidation by the dissimilatory perchlorate-reducing microorganism Azospira suillum PS. mBio 8:e2023-16. doi: $10.1128 / \mathrm{mBio} .02023-16$

Ming, D. W., Archer, P. D. Jr., Glavin, D. P., Eigenbrode, J. L., Franz, H. B., et al. (2014). Volatile and organic compositions of sedimentary rocks in Yellowknife bay, gale crater, mars. Science 343:1245267. doi: 10.1126/science.1245267

Ojha, L., McEwen, A., Dundas, C., Byrne, S., Mattson, Wray, J., et al. (2014). HiRISE observations of recurring slope lineae (RSL) during southern summer on Mars. Icarus 231, 365-376. doi: 10.1016/j.icarus.2013.12.021

Ojha, L., Wilhem, M. B., Murchie, S. L., McEwen, A. S., Wray, J. J., Hanley, J., et al. (2015). Spectral evidence for hydrated salts in recurring slope lineae on mars. Nature Geosci. 8, 829-832. doi: 10.1038/ngeo2546

Oren, A., Bardavid, R. E., and Mana, L. (2014). Perchlorate and halophilic prokaryotes: implications for life on mars. Extremophiles 18, 75-80. doi: 10.1007/s00792-013-0594-9

Ragsdale, S. W. (2004). Life with carbon monoxide. Crit. Rev. Biochem. Mol. Biol. 39,165-195. doi: 10.1080/10409230490496577

Sindoni, G., Formisano, V., and Geminale, A. (2010). Observations of water vapour and carbon monoxide in the martian atmosphere with the SWC of PFS/MEX. Planet. Space Sci. 59, 149-162. doi: 10.1016/j.pss.2010.12.006

Steele, A., McCubbin, F. M., Fries, M., Kater, L., Boctor, N. Z., Fogel, M. L., et al. (2012). A reduced organic component in martian basalts. Science 337, 212-215. doi: $10.1126 /$ science. 1220715

Stern, J. C., Sutter B., Jackson, W. A., Navarro-González, R., McKay, C. P., Ming, D. W., et al. (2017). The nitrate/(per)chlorate relationship on Mars. Geophys. Res. Lett. 44, 2643-2651. doi: 10.1002/2016GL072199

Waite, J. H., Glein, C. R., Perryman, R. S., Teolis, B. D., Magee, B. A., Miller, G., et al. (2017). Cassini finds molecular hydrogen in the Enceladus plume: evidence for hydrothermal processes. Science 356, 155-159. doi: 10.1126/science. aai8703

Waite, J. H. Jr., Lewis, W. S., Magee, B. A., Lunine, J. I., McKinnon, W. B., et al. (2009). Liquid water on Enceladus from observations of ammonia and 40Ar in the plume. Nature 460, 487-490. doi: 10.1038/nature08153

Weber, C. F., and King, G. M. (2017). Volcanic soils as sources of novel CO-oxidizing Paraburkholderia and Burkholderia: Paraburkholderia hiiakae sp. nov., Paraburkholderia metrosideri sp. nov., Paraburkholderia paradisi sp. nov., Paraburkholderia peleae sp. nov., and Burkholderia alpina sp. nov. a member of the Burkholderia cepacia complex. Front. Microbiol. 8:207. doi: 10.3389/fmicb. 2017.00207

Weber, C. F., and King, G. M. (2009). Water stress impacts on bacterial carbon monoxide oxidation on recent volcanic deposits. ISME J. 3, 1325-1334 doi: 10.1038/ismej.2009.70

Zolotov, M. Y. (2007). An oceanic composition on early and today's Enceladus. Geophys. Res. Lett. 34:L23203. doi: 10.1029/2007gl031234

Zolotov, M. Y., Shock, E. L., Barr, A. C., and Pappalardo, R. T. (2004). "Brine pockets in the icy shell of Europa: distribution, chemistry, and habitability," in Proceedings on Europa's Icy Shell: Past, Present, and Future, LPI Contribution No. 1195 (Houston, TX: Lunar and Planetary Institute), $100-101$.

Conflict of Interest Statement: The authors declare that the research was conducted in the absence of any commercial or financial relationships that could be construed as a potential conflict of interest.

Copyright (c) 2017 Myers and King. This is an open-access article distributed under the terms of the Creative Commons Attribution License (CC BY). The use, distribution or reproduction in other forums is permitted, provided the original author(s) or licensor are credited and that the original publication in this journal is cited, in accordance with accepted academic practice. No use, distribution or reproduction is permitted which does not comply with these terms. 